\title{
An exploratory study on the preparation and evaluation of a "same-day" adipose stem cell-based tissue-engineered vascular graft
}

\author{
Darren G. Haskett, PhD, ${ }^{\mathrm{a}, \mathrm{b}, \mathrm{c}}$ Kamiel S. Saleh, BS, ${ }^{\mathrm{b}}$ Katherine L. Lorentz, MS, ${ }^{\mathrm{b}, \mathrm{c}}$
}

Alexander D. Josowitz, BS, ${ }^{\mathrm{b}}$ Samuel K. Luketich, MS, ${ }^{\mathrm{b}, \mathrm{d}}$ Justin S. Weinbaum, PhD, , , , e

Lauren E. Kokai, PhD, ${ }^{\mathrm{c}, \mathrm{f}}$ Antonio D’Amore, PhD, ${ }^{\mathrm{a}, \mathrm{b}, \mathrm{c}, \mathrm{d}, \mathrm{g}}$ Kacey G. Marra, PhD, ${ }^{\mathrm{b}, \mathrm{c}, \mathrm{f}}$ J. Peter Rubin, MD, ${ }^{\mathrm{b}, \mathrm{c}, \mathrm{f}}$ William R. Wagner, $\mathrm{PhD},{ }^{\mathrm{a}, \mathrm{b}, \mathrm{c}, \mathrm{d}, \mathrm{h}}$ and David A. Vorp, $\mathrm{PhD}^{\mathrm{a}, \mathrm{b}, \mathrm{c}, \mathrm{d}, \mathrm{h}, \mathrm{i}}$

\section{ABSTRACT}

Objective: Tissue-engineered vascular grafts containing adipose-derived mesenchymal stem cells offer an alternative to small-diameter vascular grafts currently used in cardiac and lower-extremity revascularization procedures. Adiposederived, mesenchymal stem cell-infused, tissue-engineered vascular grafts have been shown to promote remodeling and vascular homeostasis in vivo and offer a possible treatment solution for those with cardiovascular disease. Unfortunately, the time needed to cultivate adipose-derived mesenchymal stem cells remains a large hurdle for tissue-engineered vascular grafts as a treatment option. The purpose of this study was to determine if stromal vascular fraction (known to contain progenitor cells) seeded tissue-engineered vascular grafts would remain patent in vivo and remodel, allowing for a "same-day" process for tissue-engineered vascular graft fabrication and implantation.

Methods: Stromal vascular fraction, obtained from adult human adipose tissue, was seeded within 4 hours after acquisition from the patient onto poly(ester urethane)urea bilayered scaffolds using a customized rotational vacuum seeding device. Constructs were then surgically implanted as abdominal aortic interposition grafts in Lewis rats.

Results: Findings revealed patency in 5 of 7 implanted scaffolds at 8 weeks, along with neotissue formation and remodeling occurring in patent tissue-engineered vascular grafts. Patency was documented using angiography and gross inspection, and remodeling and vascular components were detected using immunofluorescent chemistry.

Conclusions: A "same-day" cell-seeded, tissue-engineered vascular graft can remain patent after implantation in vivo, with neotissue formation and remodeling occurring by 8 weeks. (J Thorac Cardiovasc Surg 2018;156:1814-22)

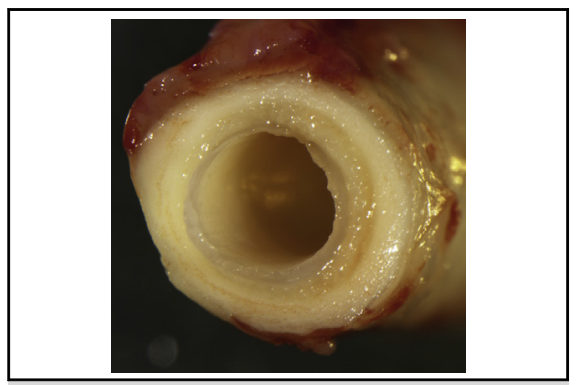

Fully remodeled TEVG after 8 weeks in vivo showing neotissue formation and patency.

\section{Central Message}

A TEVG generated within the timeframe of a single day that remodels into a functional arterial segment is feasible and represents a step toward clinical reality.

\section{Perspective}

The current field of TEVG technology still requires long fabrication times and faces regulatory hurdles. We now present work using stem cells directly sourced from human lipoaspirates for TEVG fabrication in an approach that allows cell harvest, seeding, and implantation within a 24-hour period, which is a critical step toward clinical translation.

See Editorial Commentary page 1823.
From the Departments of ${ }^{\mathrm{a} S}$ Urgery, ${ }^{\mathrm{b}}$ Bioengineering, ${ }^{\mathrm{d}}$ Chemical and Petroleum Engineering, ${ }^{\mathrm{f}}$ Plastic Surgery, ${ }^{\mathrm{i}}$ Cardiothoracic Surgery, and ${ }^{\mathrm{e}}$ Pathology, ${ }^{\mathrm{c}} \mathrm{McG}$ cowan Institute for Regenerative Medicine, and ${ }^{\mathrm{h}}$ Center for Vascular Remodeling and Regeneration,

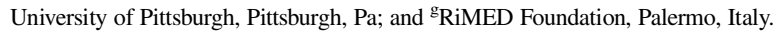

This work was supported by the National Institutes of Health (T32 HL098036 to D.G.H.; R21 EB016138, R21 HL130784, and R01 HL130077 to D.A.V.); and the RiMED Foundation (0057091 to A.D.).

Received for publication Feb 6, 2018; revisions received May 17, 2018; accepted for publication May 31, 2018; available ahead of print July 26, 2018.

Address for reprints: David A. Vorp, PhD, University of Pittsburgh, Suite 300, Center for Bioengineering (CNBIO), 300 Technology Drive, Pittsburgh, PA 15219 (E-mail: vorp@pitt.edu).

0022-5223/\$0.00

Published by Elsevier Inc. on behalf of The American Association for Thoracic Surgery

https://doi.org/10.1016/j.jtcvs.2018.05.120
Cardiovascular disease remains the leading cause of death in the United States, significantly contributing to morbidity and mortality. ${ }^{1-3}$ Treatment costs are expected to reach more than $\$ 918$ billion per year by 2020 , making it the highest total healthcare expenditure in the United States. ${ }^{4}$ Commonly

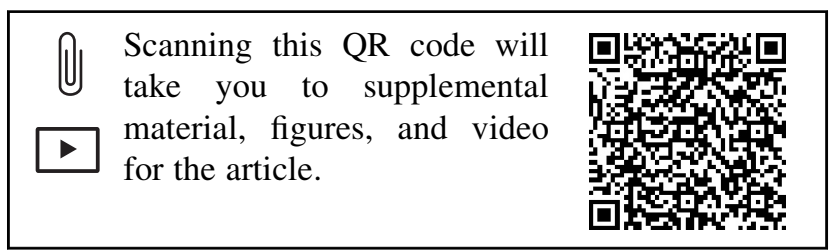




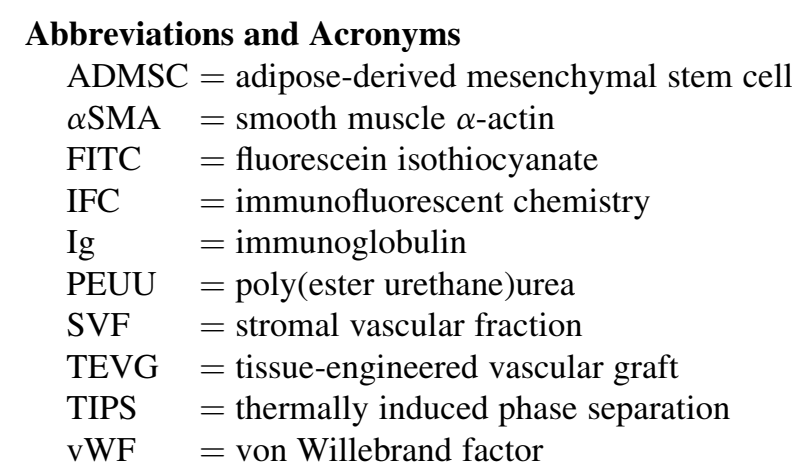

performed procedures in the treatment of cardiovascular disease include bypass graft replacement using native autologous vessels comprising the thoracic and radial arteries and saphenous vein, 2,5,6 with coronary artery bypass accounting for approximately 400,000 operations performed annually in the United States as of 2016. ${ }^{7}$ However, use of autologous vessels comes with inherent drawbacks, such as size and mechanical mismatch, availability, and complications associated with morbidity from surgical harvest. ${ }^{8-11}$ In addition, synthetic grafts fail because of acute thrombosis at diameters less than $6 \mathrm{~mm}$. $^{12-14}$

To overcome these limitations, small-diameter $(<6 \mathrm{~mm})$ tissue-engineered vascular grafts (TEVGs) have been developed that resist thrombosis and intimal hyperplasia. ${ }^{15-21}$ Much of this work uses cell-based TEVGs. However, such cell-based TEVGs come with the limitations that cells often require time to expand before TEVG fabrication, and cell seeding on the TEVG scaffold often requires lengthy bioreactor culture before implantation. Furthermore, cell culture and expansion limit clinical applicability because of regulatory concerns set by the Food and Drug Administration, which recommends the use of fresh cells with minimized time between isolation and implantation. ${ }^{22}$

A noncultured, progenitor cell-rich population known as the stromal vascular fraction (SVF) could circumvent these concerns about cell-based TEVGs. In a recent study by our group, scaffolds seeded with SVF and exposed to a 48-hour dynamic culture period produced TEVGs in vivo that were comparable to TEVGs produced using cultured adiposederived mesenchymal stem cells (ADMSCs). ${ }^{6}$ However, although the culture period and time of fabrication were greatly reduced in this study, it still used an in vitro culture step.

We hypothesized that the removal of any and all culture steps, including the 48-hour dynamic bioreactor culture period in our previously reported SVF seeded TEVG model, would not affect productive in vivo remodeling, allowing for a "same-day" process for TEVG fabrication and implantation (Video 1). To test this hypothesis, a smallscale proof of concept study was conducted to demonstrate the ability for a completely noncultured cell population seeded onto an elastomeric scaffold and immediately placed as an interpositional graft in the abdominal aorta to remodel into a functional TEVG. To relate this study to earlier work in our laboratory, we compared the phenotypic diversity of cells within SVF seeded directly into our TEVGs for immediate implantation with that within our TEVGs after a 48hour dynamic culture period, as well as with that of SVF that is culture expanded before seeding onto our TEVGs. Our results demonstrate that TEVGs manufactured in a single day perform similar to cultured TEVGs or TEVGs seeded with culture-expanded cells, representing a significant advancement in TEVG clinical translation.

\section{MATERIALS AND METHODS \\ Patient Selection and Stromal Vascular Fraction Isolation}

SVF was obtained from the discarded waste adipose tissue of nondiabetic female human adults, 45 years of age or younger, undergoing elective standard of care liposuction, abdominoplasty, or panniculectomy procedures. Tissue was transferred to the research team through an honest broker under University of Pittsburgh Institutional Review Board exempt protocol \#0511186. ${ }^{23,24}$ Because this was an exempt protocol and no identifiable information was available to the research staff, informed consent was not obtained. Patient information was limited to age in years, gender, body mass index, and diabetic status.

SVF was freshly isolated using previously described methods. ${ }^{25,26}$ Briefly, adipose tissue ( $\sim 250 \mathrm{~mL}$ per patient) was cut into approximately $10-\mathrm{mL}$ portions that were each placed into separate $50-\mathrm{mL}$ conical tubes Each piece was minced and added to a collagenase solution, Hanks' Balanced Salt Solution (Invitrogen, Carlsbad, Calif), containing 3.5\% bovine serum albumin (Millipore, Charlottesville, Va) and $1 \mathrm{mg} / \mathrm{mL}$ collagenase type II (Worthington, Lakewood, NJ). ${ }^{26}$ Tubes were then incubated at $37^{\circ} \mathrm{C}$ with agitation for approximately 1 hour. Digested tissue was filtered through successive $425-$ and $180-\mu \mathrm{m}$ sieves (WS Tyler, Mentor, Ohio) to remove undigested pieces and then centrifuged at $1000 \mathrm{rpm}$ for 10 minutes at ambient temperature. After centrifugation, the cell pellet was resuspended in an $\mathrm{NH}_{4} \mathrm{Cl}$-based buffer (Beckman Coulter, Miami, Fla; Cat No. IM3630d) to lyse red blood cells. The resulting cell suspension was filtered once more through a $180-\mu \mathrm{m}$ sieve and centrifuged (1000 rpm, 10 minutes, ambient temperature). The resulting pellet, termed "SVF," was resuspended in Hanks' Balanced

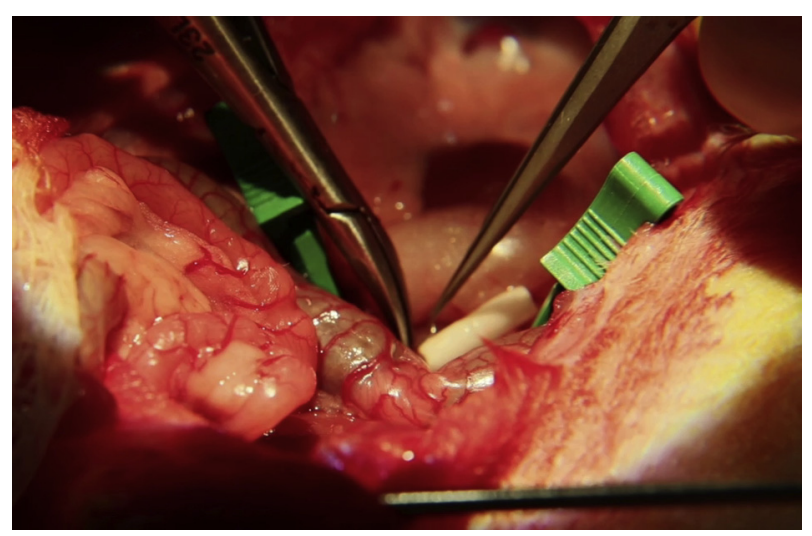

VIDEO 1. Video walkthrough of the "same-day" process of vascular graft fabrication and implantation explored in this article. Video available at: https://www.jtcvs.org/article/S0022-5223(18)31753-7/fulltext. 
Salt Solution and maintained on ice for up to 2 hours until scaffold seeding or cell culture. The process typically yielded 30 to 40 million fresh SVF cells when using $200 \mathrm{~mL}$ of adipose tissue. Freshly isolated SVF was used for culture or immediately seeded onto our TEVG scaffolds for analysis or immediate implantation.

\section{Scaffold Fabrication}

Scaffolds used in this study were fabricated out of poly(ester urethane) urea (PEUU), which is biodegradable and elastomeric. Scaffolds were bilayered, tubular, and approximately $1 \mathrm{~cm}$ long, and had an approximately 1.3-mm inner diameter to approximate the shape and size of a rat infrarenal abdominal aorta. Scaffolds were manufactured and used as described previously. ${ }^{16,17,27,28}$ Briefly, PEUU polymer was used to fabricate both layers of the scaffold. The porous inner layer of the scaffold was created using thermally induced phase separation (TIPS) in a tubular mold. The TIPS layer was then coated by electrospinning an additional layer of PEUU for mechanical stability. This scaffold has already shown efficacy in the TEVG context using multiple cell types. $6,17,19,27-29$

\section{Cell Culture and Stromal Vascular Fraction Expansion for Phenotypic Analysis}

A fraction of SVF was expanded in cell culture using defined culture media (1:1 Dulbecco's modified Eagle's medium \#11965; Gibco, Waltham, Mass) with Dulbecco's modified Eagle's medium/F12 (\#113300; Gibco) and $10 \%$ fetal bovine serum (\#S11550; Atlanta Biologicals, Flowery Branch, Ga), antibiotics (1\% penicillin/streptomycin, 0.5\% Fungizone, $0.1 \%$ gentamycin; all from Gibco), and $10 \mu \mathrm{L} / \mathrm{L}$ dexamethasone mixed with 25\% Preadipocyte Growth Medium (\#C-27410, \#C-39425; PromoCell, Heidelberg, Germany). Upon reaching $80 \%$ confluency, the cells were removed from the plate by trypsinization and termed "passage 0 ADMSC." These cells were then plated for additional expansion up to passage 4 using the same protocol or used directly for seeding experiments.

\section{Scaffold Seeding and Incubation}

Scaffolds were seeded with SVF or passage 0 to 4 ADMSC using a customized rotational vacuum seeding device as described and used previously. ${ }^{19,29-31}$ Briefly, the scaffolds were mounted within the device, and the cell suspension was infused with vacuum and rotation (flow rate of $1 \mathrm{~mL} / \mathrm{min}$, vacuum pressure of $-120 \mathrm{~mm} \mathrm{Hg}$, and rotation speed of $15 \mathrm{rpm}$ ). Each scaffold was seeded with approximately 3 million SVF cells.

After seeding, the constructs were placed in defined culture media (see "Cell Culture and Stromal Vascular Fraction Expansion for Phenotypic Analysis") for transport and immediate implantation (see "Aortic Implantation of Seeded Scaffolds") or given 4 hours incubation for phenotypic analysis, allowing the cells to adhere to the scaffold under static conditions. At this point, constructs were fixed or dynamically cultured for an additional 48 hours. As previously described, dynamic culture entailed suspension in a 500-mL spinner flask (Kontes \#Cytostir 882911-0250) $\left(100 \mathrm{~mL}\right.$ of defined culture media, rotation speed $15 \mathrm{rpm}$ ). ${ }^{6,16,25}$

\section{Phenotypic Analysis of Seeded Scaffolds}

Seeded constructs used for phenotypic analysis were fixed in $4 \%$ paraformaldehyde, washed, placed in a $30 \%$ sucrose solution for 30 minutes, and frozen at $-20 \mathrm{C}$. Frozen seeded constructs were then sectioned using a cryostat at a thickness of $10 \mu \mathrm{m}$ onto gelatin-coated slides. To determine the SVF cell populations adhering to the PEUU scaffolds, immunofluorescent chemistry (IFC) was performed. Sections of the seeded constructs were permeabilized using a $0.1 \%$ Triton solution, and $5 \%$ goat serum was used for blocking. Sections were incubated with 1:100 dilutions of rabbit anti-human CD90 (ab133350, Abcam, Cambridge, UK), rabbit anti-human CD31 (ab28364, Abcam), or rabbit anti-human CD34 (ab81289, Abcam) to evaluate the presence of mesenchymal stem cell, endothelial cell, or endothelial progenitor cell populations, respectively. Additional IFC for SVF markers CD14, CD45, and CD105 was performed (Figure E1). All IFC staining included a primary delete for a negative control and an additional isotype control (ab172730, Abcam). Seeded constructs were then incubated with 1:1000 dilutions of FITC-conjugated goat anti-rabbit immunoglobulin (Ig)-G secondary antibody (611-1202, Rockland, Inc, Pottstown, Pa) and then counterstained with DAPI (B2883, Sigma-Aldrich, St Louis, Mo) to mark cell nuclei. Imaging was conducted on a Nikon Eclipse 90i microscope (Nikon, Tokyo, Japan).

Cell populations were quantified by calculating the ratio between cells exhibiting each specific cell marker (CD90, CD31, and CD34) and the total cell count as established by evaluating the DAPI stained nuclei; a custom ImageJ macro was built for this purpose (for greater detail, see Supplemental Material: ImageJ Macro Analysis).

\section{Aortic Implantation of Seeded Scaffolds}

All animal procedures were performed under a protocol approved by the University of Pittsburgh Institutional Animal Care and Use Committee. Seeded scaffolds were surgically implanted as abdominal aortic interpositional grafts in Lewis rats as described previously. ${ }^{17,27,28}$ Briefly, rats were anesthetized using ketamine $(30 \mathrm{mg} / \mathrm{kg})$ and placed in a supine position and kept under sedation for the duration of the surgery using nose cone administration of isoflurane (induction $[2 \%-5 \%]$ and maintenance $[0.25 \%$ $4 \%$ ] $1.5 \mathrm{~L} / \mathrm{min}$ in oxygen). An incision was made in the abdominal wall, and the infrarenal abdominal aorta was exposed through blunt dissection. Microclamps were applied to the aorta, which was then bisected, upon which a 1$\mathrm{cm}$ seeded construct was placed interpositionally and sutured with 10-0 Prolene (Ethicon, Somerville, NJ) (Figure E2). After the graft was secured, the clamps were released and the patency was verified by observation of the distal pulse pressure. The animals were then closed with 3-0 polyglactin sutures (McKesson, Richmond, Va). Buprenorphine hydrochloride was administered postoperatively every 12 hours for the first 72 hours, and animals were maintained on an anticoagulant (dipyridamole, aspirin) schedule for 4 weeks, as previously described. ${ }^{16}$ Implanted scaffolds were allowed to remodel for 8 weeks to allow enough time for remodeling and neotissue formation to occur as previously observed. ${ }^{6,16,29,30}$ Rats were then killed using isoflurane (induction $[2 \%-5 \%] 1.5 \mathrm{~L} / \mathrm{min}$ oxygen) and a single intracardiac injection of heparin $/ \mathrm{KCl}$. The descending aorta proximal to the TEVG was catheterized and injected with $\mathrm{x}$-ray contrast agent for imaging, and an angiography was performed to determine in vivo patency. The TEVG and adjacent aortic tissue were excised for gross inspection and further analysis.

\section{Immunofluorescent and Histologic Evaluation}

Explanted TEVGs were fixed in $4 \%$ paraformaldehyde before frozen sectioning or paraffin embedding. Frozen sectioning was performed as described earlier using fluorescein isothiocyanate (FITC)-conjugated mouse anti-von Willebrand factor (vWF - 1:250; V2700-07, US Biological, Salem, Mass) and mouse anti-smooth muscle $\alpha$-actin $(\alpha$ SMA) (A5228, Sigma-Aldrich) along with a TRITC conjugated anti-mouse IgG secondary antibody (ab6786, Abcam). The presence of any remaining implanted cells was also determined using IFC for rabbit anti-human CD90 (ab133350, Abcam) and human nuclear antigen (MAB1281, MEMD, Millipore) with FITC-conjugated goat anti-rabbit IgG secondary antibody (611-1202, Rockland Inc) and counterstained with DAPI (B2883, Sigma-Aldrich) to mark cell nuclei.

Histology was performed on paraffin-embedded sections of both explanted TEVGs and native rat aorta proximal to the anastomosis using the Histology Core at the McGowan Institute for Regenerative Medicine. Sections were stained for Verhoeff van Gieson staining to indicate elastin fibers and Masson's trichrome to indicate collagen and other vascular components.

\section{Statistical Analysis}

A 2-way analysis of variance was performed on the phenotypic analysis data using SPSS software (IBM, Armonk, New York, NY) between the different cell markers and each passage of cells. A Bonferroni confidence 
TABLE 1. Stromal vascular fraction donor information, along with collection and procedure information

\begin{tabular}{llllll}
\hline Donor ID No. & Gender & Age, $\mathbf{y}$ & Procedure & Use & Result of implantation \\
\hline HS 1 & Female & 45 & Panniculectomy & IFC and culture & Not implanted \\
HS 2 & Female & 27 & Panniculectomy & IFC and culture & Not implanted \\
HS 3 & Female & 20 & Panniculectomy & IFC and culture & Not implanted \\
HS 4 & Female & 35 & Liposuction & IFC and culture & Not implanted \\
HS 5 & Female & 41 & Abdominoplasty & IFC and culture & Not implanted \\
HS 6 & Female & 44 & Panniculectomy & IFC and culture & Not implanted \\
HS 7 & Female & 28 & Panniculectomy & Implant & 1 occluded (SVF-1), \\
& & & & If fully remodeled (SVF-2) \\
HS 8 & Female & 42 & Liposuction & Implant & 2 occluded (SVF-3) \\
HS 9 & Female & 33 & Panniculectomy & Implant & 2 fully remodeled \\
HS 10 & Female & 25 & Panniculectomy & (SVF-6 and -7) \\
\hline
\end{tabular}

Use refers to use in cellular culture and IFC comparisons with uncultured seeded scaffolds or use in "same-day" implant procedures and explant results. IFC, Immunofluorescen chemistry; $S V F$, stromal vascular fraction.

interval adjustment was made, and pairwise comparisons were made post hoc. In addition, a paired Student $t$ test was used to evaluate statistical differences in cell markers between immediate and 48-hour cultured constructs.

\section{RESULTS}

\section{Phenotypic Analysis of Stromal Vascular Fraction and Passaged Adipose-Derived Mesenchymal Stem Cells}

SVF was gathered from 6 different subjects at the Adipose Stem Cell Research Laboratory - University of Pittsburgh (available patient information provided in Table 1). IFC analysis of the seeded SVF/ADMSC phenotypes showed a distinct shift with increasing passage (Figure 1). Specifically, scaffolds seeded with culture expanded cells were found to have an approximately 4-fold increase in CD90 positive staining, whereas the percentage of cells positive for CD31 and CD34 became undetectable by passage $4(P=.004)$.

Additional evaluations were made comparing scaffolds seeded with fresh, uncultured SVF that were fixed immediately after seeding versus scaffolds that had 48 hours of dynamic culture before being fixed. Adherent cells in constructs seeded with fresh, uncultured SVF displayed markers for CD90, CD31, and CD34 in varying amounts with CD31 positive cells significantly higher than CD90 positive cells in the fresh $\operatorname{SVF}(P=.023)$. Additional IFC analysis comparing the fresh, uncultured SVF found no difference in $\mathrm{CD}$ marker expression between constructs given only a brief time for cells to adhere to the scaffold (to compare with constructs used for implantation) and constructs given a 48-hour dynamic culture period (the standard culture used in previous studies ${ }^{6,16,30}$ ) (Figure 2).

\section{"Same-Day" Tissue-Engineered Vascular Graft Implantation and Remodeling}

A total of 7 "same-day" implantation procedures, from 4 different SVF collections, were performed for this study, with most SVF harvest procedures resulting in 2 implanted scaffolds each (Table 1). Patency was observed in 5 of the 7 TEVG explants through angiography (Figure 3,A) and gross inspection, which classified explants as fully remodeled (Figure 3,B), partially remodeled (Figure 3, $C$ ), or occluded (Figure 3,D) (Supplemental Material: Remodeling Classifications). Patent explanted "sameday" TEVGs indicated remodeling into a vascular-like tissue after 8 weeks of implantation in vivo, although 2 showed only partial remodeling (Table 1). Of the 2 TEVGs that were occluded, intimal hyperplasia was observed at both the proximal and distal anastomoses. No thrombus was found within either of the partially remodeled grafts or the occluded grafts, suggesting that blood was able to pass freely though the conduit before the anastomotic hyperplasia emerged.

Broadly, patency corresponded with a layer of neotissue that formed on top of the TIPS layer, surrounding an open lumen. Further IFC analysis of the patent grafts revealed extensive $\mathrm{vWF}$ staining along the luminal edge in a continuous manner, indicating the presence of an endothelial lining and seemingly organized $\alpha$ SMA staining within the neotissue in 3 of the fully remodeled patent grafts (Figure 4, $A$ and $B$ ). The other 2 partially remodeled grafts lacked an organized luminal lining or vascular-like tissue formation (Figure 4, $C$ and $D$ ) compared with a native rat aorta (Figure 4, $E$ and $F$ ). Further histologic analysis of the fully remodeled TEVGs using Verhoeff van Gieson staining also revealed elastin fibers within the neotissue especially toward the luminal edge, and Masson's trichrome staining revealed the presence of newly formed collagen (Figure 5).

\section{DISCUSSION}

This study showed that adherent cells in the SVF shifted their surface markers away from endothelial and endothelial 

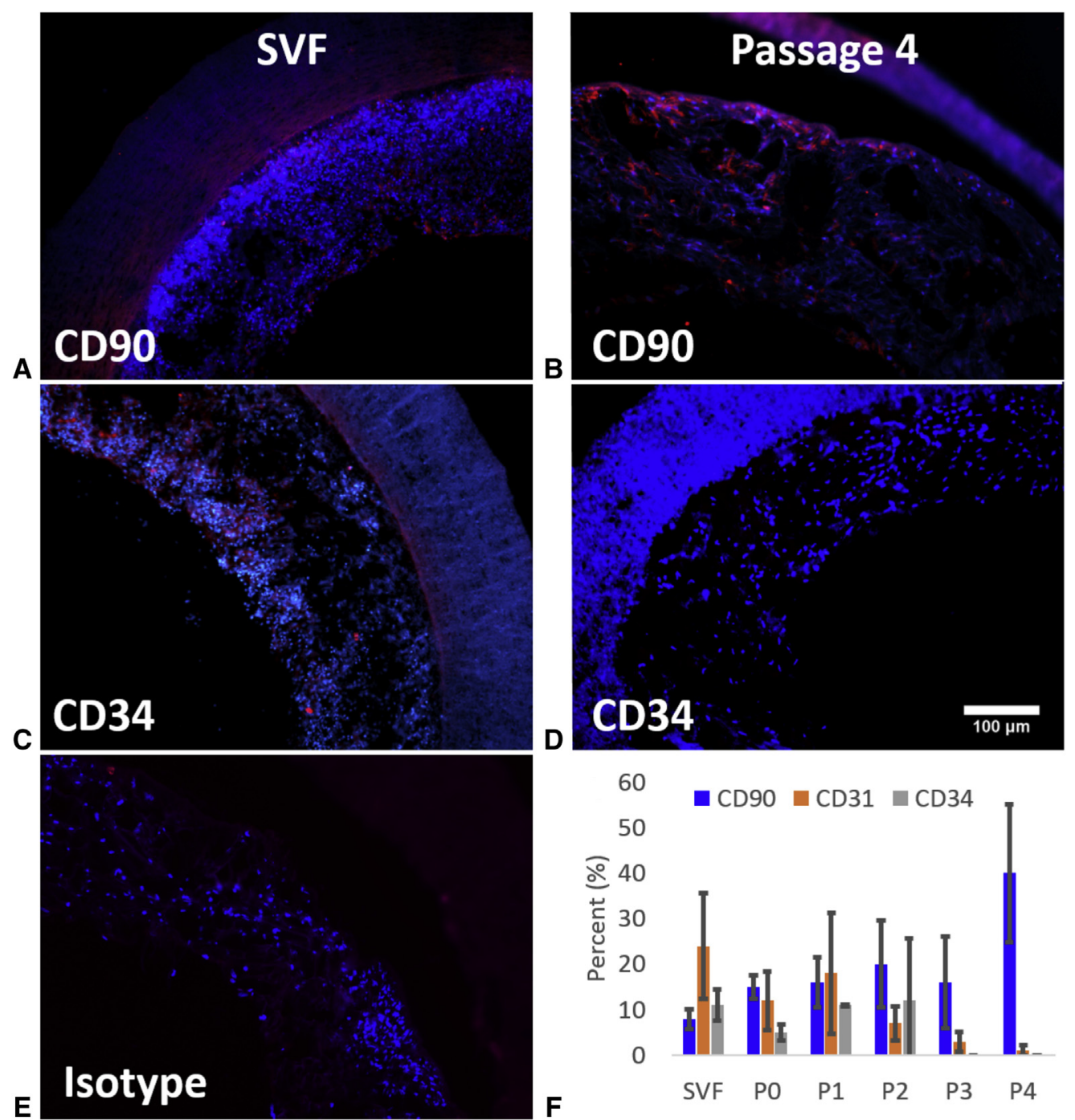

FIGURE 1. Representative IFC images depicting positive staining of cells for CD90 (A and B) and CD34 (C and D). Initial SVF cells stained positively for both CD90 (A) and CD34 (C), whereas positive staining for CD90 (B) increased and positive staining for CD34 (D) decreased at passage 4. E, Isotype control demonstrating no nonspecific immunopositive cells. F, IFC analysis of percentage of adherent cells positive for cell markers CD90 (mesenchymal stem cell marker), CD31 (endothelial marker), and CD34 (endothelial progenitor marker) seeded onto PEUU scaffolds with cells from fresh SVF out to passage 0 through 4 and given a 48 -hour dynamic culture period. SVF, Stromal vascular fraction.

progenitor markers (CD31 and CD34) and toward expression of the ADMSC marker CD90 by passage 4 . Furthermore, the adherent cells in the SVF had the same phenotypic distribution with or without the previously described 48-hour dynamic culture period. ${ }^{6}$ Most important, removal of the 48-hour dynamic culture period (or any culture other than a brief period to allow cells to adhere to the scaffolds) and the implementation of the collection, isolation, seeding, and implantation steps within a 24-hour, "same-day" procedure generated a functioning TEVG. With streamlining, the entire procedure was able to be accomplished within a 12-hour period by the end of the study.

The focus of work in our laboratory, and the motivation for this study, is the generation of a TEVG within a clinically relevant time scale. Previous work by Krawiec and colleagues ${ }^{16,25}$ made use of cultured ADMSCs that took up to 1 month or more from collection to implantation. This waiting time between collection and implantation was able to be drastically reduced when using a noncultured cell source, but still included dynamic culture, thus limiting its clinical feasibility. When comparing the "same-day" procedure with that of SVF-seeded constructs given 48 hours of dynamic culture, patency rates were similar. ${ }^{6}$ Notably, none of the "sameday" TEVGs failed because of thrombosis. A recent review suggests that TEVG failure due to thrombosis is caused by the graft having poor blood interaction, whereas in contrast, failure due to hyperplasia often can be attributed to conduit mismatch, surgical error, or damage to the endothelium that creates hyperplastic conditions. ${ }^{2}$ Previously, bare PEUU 


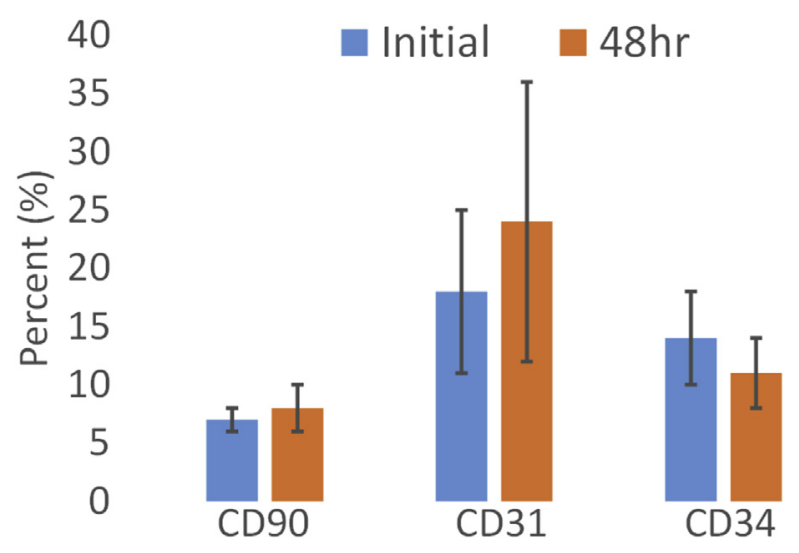

FIGURE 2. Average cell populations for SVF constructs fixed after initial incubation and 48 hours of dynamic culture $(\mathrm{n}=6)$. A paired $t$ test indicated no significant statistical difference between initial and 48-hour constructs for each of the CD90, CD31, and CD34 markers $(P=.63, .68$, and .46 , respectively).

scaffolds have been used with patency rates of less than $40 \%,{ }^{19}$ whereas other work in our laboratory has shown patent TEVGs can be generated using bone marrow-derived MSCs, ${ }^{17}$ muscle-derived $\mathrm{MSCs},{ }^{27}$ and pericytes ${ }^{28}$ with varying patency rates, whereas patency rates of $100 \%$ have been achieved with ADMSCs from young, nondiabetic donors. ${ }^{16}$ Thus, it is important to note that although only 5 of the 7 TEVGs were patent at 8 weeks, a microsurgeon highly experienced with the model would likely experience higher patency rates.

Several other groups have moved a TEVG toward clinical implementation. Shinoka ${ }^{32}$ and Breuer have tested their technology as low-pressure conduits in large animal models and human trials, ${ }^{33,34}$ whereas L'Heureux and colleagues at Cytograft (Novato, Calif) developed a TEVG constructed using a cell sheet technology as an arteriovenous shunt for hemodialysis under the product name Lifeline. ${ }^{35}$ Of note, the Lifeline technology used a patient's own fibroblasts and endothelial cells in 2 rounds of culture that took up to 7 months to complete, although newer methods have moved into human trials with devitalized scaffolds lacking an endothelium. ${ }^{21}$ Niklason and colleagues at Humacyte (Morrisville, NC) have established the "human acellular vessel" line of TEVG products. ${ }^{36}$ This TEVG is fabricated using bioreactor culture and can take 1 month to fabricate. ${ }^{32}$ Although these TEVGs have already made it to clinical trials, the time needed for bioreactor
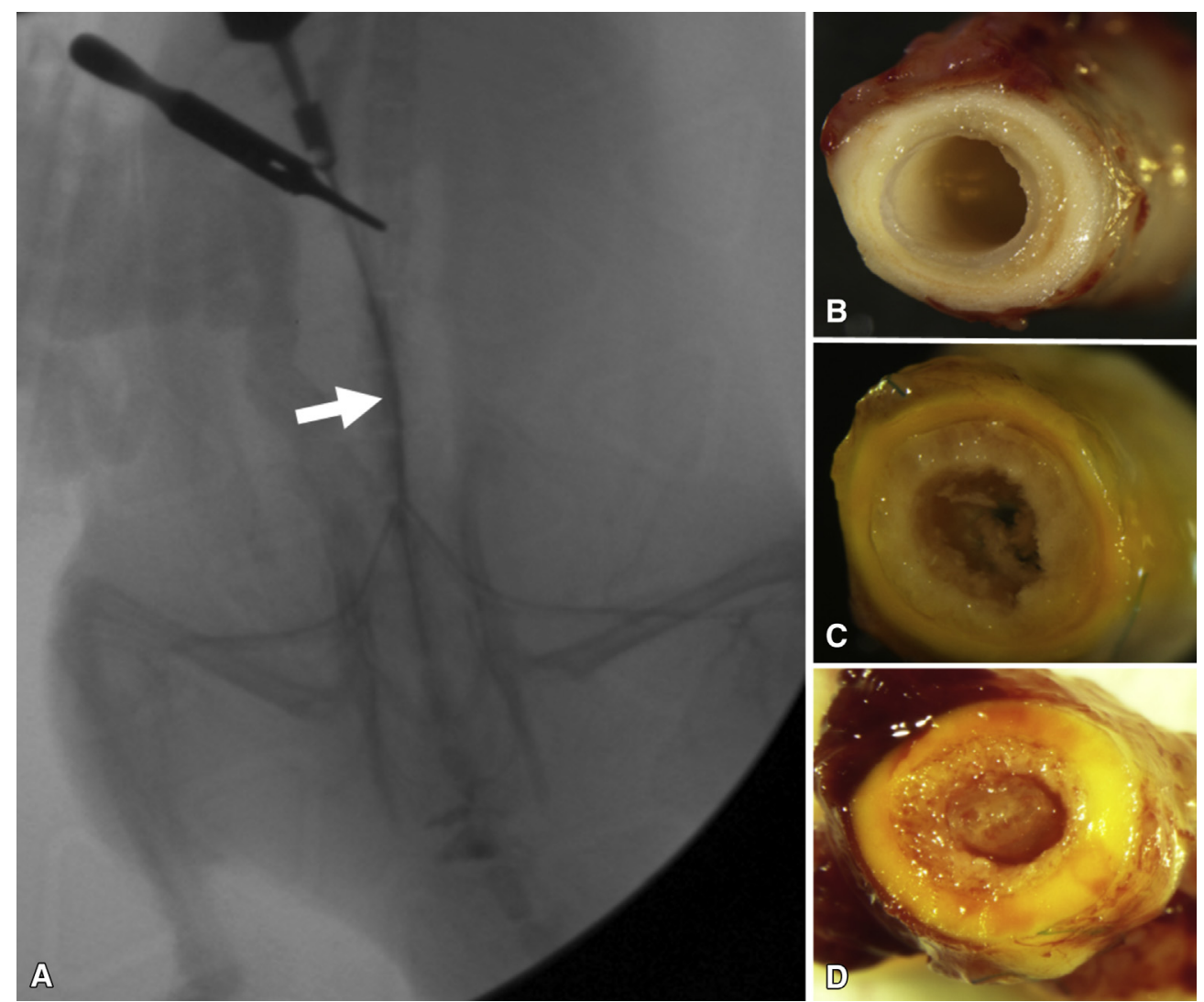

FIGURE 3. A, Angiogram confirming flow through a representative (SVF-2 in Table 1) fully remodeled, SVF-seeded TEVG (arrow) 8 weeks after implantation. B-D, Gross inspection of explanted TEVGs revealing patency and neotissue formation for a fully remodeled TEVG (B, SVF-6), partially remodeled patent TEVG (C, SVF-5), and an occluded TEVG (D, SVF-3). 


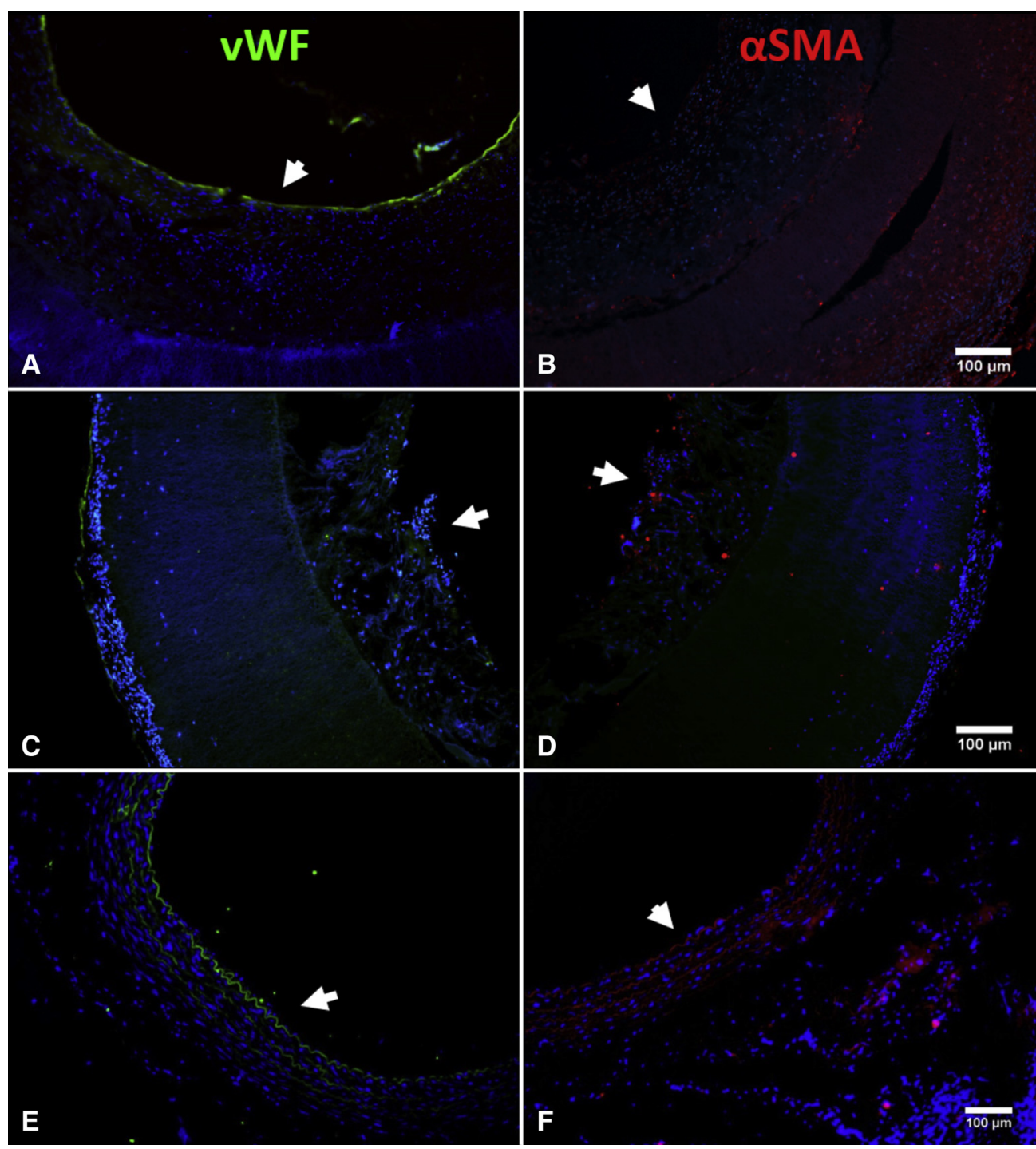

FIGURE 4. A and B, Staining for vWF (EC marker) (left) and $\alpha$ SMA (SMC marker) (right) for a fully remodeled TEVG (SVF-2) indicating a complete endothelial lining and the presence of SMCs. C and D, Staining for vWF (left) and $\alpha$ SMA (right) for a patent but not fully remodeled TEVG (SVF-5) indicating presence of some endothelial-like cells and some cells expressing $\alpha$ SMA but not a full endothelial lining or vascular-like structures within the neotissue that was found in the fully remodeled TEVGs. E and F, Staining for vWF (left) and $\alpha$ SMA (right) for a native rat aorta for comparison with native tissue. Arrows indicate the lumen. Counterstained with DAPI (blue - cell nuclei). $v W F$, von Willebrand factor; $\alpha S M A$, smooth muscle $\alpha$-actin.

incubation, with potential cellular transformation or contamination, and an indeterminate shelf life of both the Cytograft and Humacyte TEVGs may raise some concern and possibly limit their clinical utility. ${ }^{21,36}$ Thus, the impetus remains for a scaffold that can be safely stored long term and then seeded and implanted as a TEVG within a single operating room.

\section{Study Limitations}

Future work will have to go beyond a preliminary study performed in an immunotolerant rat model implanted with xenogeneic human cells and move toward a more clinically relevant model. This should include a large animal model to accommodate a longer and larger caliber TEVG fabricated with autologous or allogeneic cells. Such large animal models would be able to answer critical questions, such as the robustness of host cell infiltration into a longer graft. This study, like nearly all other studies done previously using TEVGs in an animal model, ${ }^{16,37}$ made use of only young animals. Going forward, a more clinically relevant recipient population must be used including older animals and nonimmunotolerant hosts. It might also be valuable to examine more closely the mechanisms of remodeling and prevention of acute thrombosis of the implant. Another limitation of our study was the amount of time and effort required to manually process enough SVF cells for seeding the TEVG. However, devices and methods to more rapidly produce SVF cells in large volumes have 


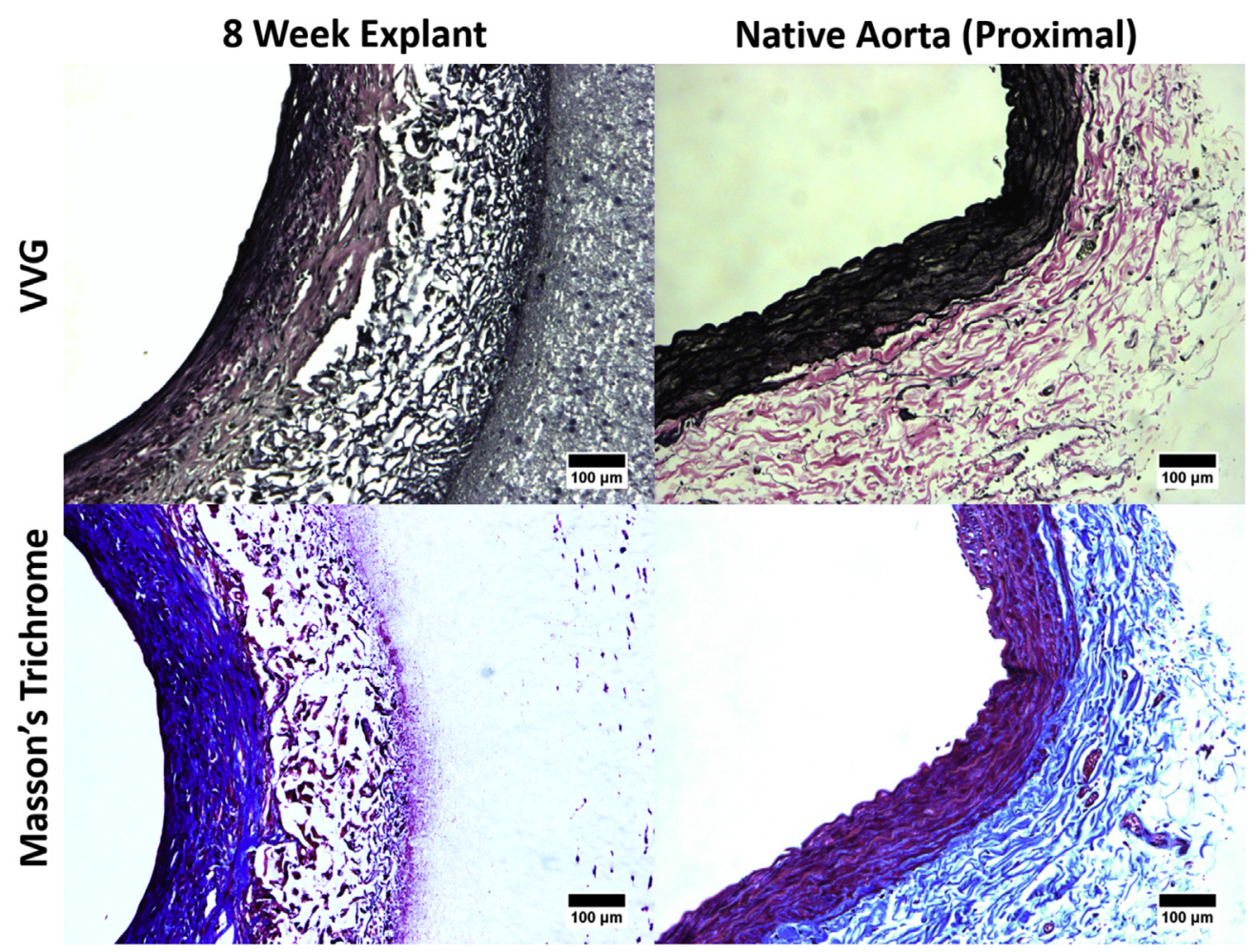

FIGURE 5. Histologic staining of fully a remodeled TEVG for Verhoeff van Gieson (top) indicating elastin (black) and Masson's trichrome (bottom) indicating collagen (blue) deposition within the TEVGs (left) compared with native rat aorta (right). VVG, Verhoeff van Gieson.

been advancing rapidly. Devices such as the Tissuegenesis ICELLATOR (Honolulu, Hawaii), GID SVF-1 (GID Europe, London, United Kingdom), Puregraft (Puregraft, Solana Beach, Calif), and Duografter II (Proteal, Barcelona, Spain) $)^{38}$ are already being used clinically and provide a feasible method to accelerate TEVG fabrication with SVF. Last, a potential limitation of implanting a construct seeded with SVF is that these cells represent a phenotypically diverse population that could differentiate in an uncontrolled manner. However, the cells are not expected to remain in the construct for long, ${ }^{39}$ and any manipulation of the SVF (eg, purification to specific cell types) would defeat the purpose of the "same-day" strategy by restoring regulatory concerns. Moreover, the SVF cell population has recently been shown to have possibly better pro-remodeling effects than cultured ADMSCs, ${ }^{24,40}$ and thus is another important facet of this model that is of current interest for further study.

This was a small-scale feasibility study demonstrating that a culture-free, single-day process could be successful and comparable to our standard ADMSC-seeded TEVG model, with phenotypic comparisons between the 2 cell populations. The motivation behind the study-to create a "same-day" TEVG-moves toward a clinically relevant technology in which an off-the-shelf scaffold could be seeded and implanted within a practical timeframe. Future directions must also answer what the long-term outcomes (beyond 8 weeks) are for such technology and if there is any phenotypic difference between donor populations that might contribute or detract from overall TEVG remodeling and maturation. Even so, the ability to generate and implant a TEVG within a single day that remodels and generates a vessel-like conduit marks significant progress. Thus, the overall goal of our work toward creating a functional TEVG by removing any culture period and streamlining the process of cell population collection, fabrication and seeding, and implantation to within a "same-day" period was met. With this and other techniques in hand, novel grafts and methods could someday make TEVGs a clinical reality.

\section{CONCLUSIONS}

Patency of our SVF-seeded TEVGs after 8 weeks in vivo was achieved using a "same-day" process. The seeded SVF cell population had no phenotypic differences between those with or without a 48-hour dynamic culture period, but was significantly different from ADMSCs at passage 4. TEVGs were able to remodel in vivo with gross inspection and histology indicating neotissue formation and immunostaining for $\mathrm{vWF}$ and $\alpha$ SMA demonstrating the presence of an endothelium and smooth muscle cells, respectively. 


\section{Conflict of Interest Statement}

Authors have nothing to disclose with regard to commercial support.

\section{References}

1. Mathers CD, Loncar D. Projections of global mortality and burden of disease from 2002 to 2030. PLoS Med. 2006;3:e442.

2. Pashneh-Tala S, MacNeil S, Claeyssens F. The tissue-engineered vascular graftpast, present, and future. Tissue Eng Part B Rev. 2016;22:68-100.

3. Zoghbi WA, Duncan T, Antman E, Barbosa M, Champagne B, Chen D, et al. Sustainable development goals and the future of cardiovascular health. A statement from the global cardiovascular disease taskforce. Eur Heart J. 2014;35:3238-9.

4. Benjamin EJ, Blaha MJ, Chiuve SE, Cushman M, Das SR, Deo R, et al; American Heart Association statistics C and stroke statistics S. Heart disease and stroke statistics-2017 update: a report from the American Heart Association. Circulation. 2017;135:e146-603.

5. Dimitrievska S, Niklason LE. Historical perspective and future direction of blood vessel developments. Cold Spring Harb Perspect Med. 2018;8:a025742.

6. Krawiec JT, Liao HT, Kwan LL, D’Amore A, Weinbaum JS, Rubin JP, et al. Evaluation of the stromal vascular fraction of adipose tissue as the basis for a stem cell-based tissue-engineered vascular graft. J Vasc Surg. 2017;66:883-90.e1.

7. Alexander JH, Smith PK. Coronary artery bypass grafting. N Engl J Med. 2016; 375:e22.

8. Athanasiou T, Saso S, Rao C, Vecht J, Grapsa J, Dunning J, et al. Radial artery versus saphenous vein conduits for coronary artery bypass surgery: forty years of competition-which conduit offers better patency? A systematic review and meta-analysis. Eur J Cardiothorac Surg. 2011;40:208-20.

9. Cho KR, Kim JS, Choi JS, Kim KB. Serial angiographic follow-up of grafts one year and five years after coronary artery bypass surgery. Eur J Cardiothorac Surg. 2006;29:511-6.

10. Goldman S, Zadina K, Moritz T, Ovitt T, Sethi G, Copeland JG, et al. Long-term patency of saphenous vein and left internal mammary artery grafts after coronary artery bypass surgery: results from a Department of Veterans affairs cooperative study. J Am Coll Cardiol. 2004;44:2149-56.

11. Masden DL, Seruya M, Higgins JP. A systematic review of the outcomes of distal upper extremity bypass surgery with arterial and venous conduits. J Hand Surg Am. 2012;37:2362-7.

12. Herring MB, Dilley R, Jersild RA Jr, Boxer L, Gardner A, Glover J. Seeding arterial prostheses with vascular endothelium. The nature of the lining. Ann Surg. 1979; 190:84-90.

13. Graham LM, Burkel WE, Ford JW, Vinter DW, Kahn RH, Stanley JC. Immediate seeding of enzymatically derived endothelium in Dacron vascular grafts. Early experimental studies with autologous canine cells. Arch Surg. 1980;115:1289-94.

14. Deutsch M, Meinhart J, Zilla P, Howanietz N, Gorlitzer M, Froeschl A, et al. Long-term experience in autologous in vitro endothelialization of infrainguinal ePTFE grafts. J Vasc Surg. 2009;49:352-62.

15. Gui L, Boyle MJ, Kamin YM, Huang AH, Starcher BC, Miller CA, et al. Construction of tissue-engineered small-diameter vascular grafts in fibrin scaffolds in 30 days. Tissue Eng Part A. 2014;20:1499-507.

16. Krawiec JT, Weinbaum JS, Liao HT, Ramaswamy AK, Pezzone DJ, Josowitz AD, et al. In Vivo functional evaluation of tissue-engineered vascular grafts fabricated using human adipose-derived stem cells from high cardiovascular risk populations. Tissue Eng Part A. 2016;22:765-75.

17. Nieponice A, Soletti L, Guan J, Hong Y, Gharaibeh B, Maul TM, et al. In vivo assessment of a tissue-engineered vascular graft combining a biodegradable elastomeric scaffold and muscle-derived stem cells in a rat model. Tissue Eng Part A. 2010;16:1215-23.

18. Prichard HL, Manson RJ, DiBernardo L, Niklason LE, Lawson JH, Dahl SL. An early study on the mechanisms that allow tissue-engineered vascular grafts to resist intimal hyperplasia. J Cardiovasc Transl Res. 2011;4:674-82.

19. Soletti L, Nieponice A, Hong Y, Ye SH, Stankus JJ, Wagner WR, et al. In vivo performance of a phospholipid-coated bioerodable elastomeric graft for small-diameter vascular applications. J Biomed Mater Res A. 2011;96: 436-48.

20. Syedain ZH, Meier LA, Lahti MT, Johnson SL, Tranquillo RT. Implantation of completely biological engineered grafts following decellularization into the sheep femoral artery. Tissue Eng Part A. 2014;20:1726-34.
21. Wystrychowski W, McAllister TN, Zagalski K, Dusserre N, Cierpka L, L'Heureux N. First human use of an allogeneic tissue-engineered vascular graft for hemodialysis access. J Vasc Surg. 2014;60:1353-7.

22. McAllister TN, Audley D, L'Heureux N. Autologous cell therapies: challenges in US FDA regulation. Regen Med. 2012;7:94-7.

23. Bliley JM, Satish L, McLaughlin MM, Kling RE, Day JR, Grahovac TL, et al. Imaging the stromal vascular fraction during soft-tissue reconstruction. Plast Reconstr Surg. 2015;136:1205-15.

24. Kokai LE, Traktuev DO, Zhang L, Merfeld-Clauss S, DiBernardo G, Lu H, et al. Adipose stem cell function maintained with age: an intra-subject study of longterm cryopreserved cells. Aesthet Surg J. 2017;37:454-63.

25. Krawiec JT, Weinbaum JS, St. Croix CM, Phillippi JA, Watkins SC, Rubin JP, et al. A cautionary tale for autologous vascular tissue engineering: impact of human demographics on the ability of adipose-derived mesenchymal stem cells to recruit and differentiate into smooth muscle cells. Tissue Eng Part A. 2014;21: 426-37.

26. Zimmerlin L, Donnenberg VS, Pfeifer ME, Meyer EM, Peault B, Rubin JP, et al. Stromal vascular progenitors in adult human adipose tissue. Cytometry A. 2010; 77:22-30.

27. He W, Nieponice A, Hong Y, Wagner W, Vorp D. Rapid engineered small diameter vascular grafts from smooth muscle cells. Cardiovasc Eng Tech. 2011;2: 149-59.

28. He W, Nieponice A, Soletti L, Hong Y, Gharaibeh B, Crisan M, et al. Pericyte-based human tissue engineered vascular grafts. Biomaterials. 2010; 31:8235-44.

29. Soletti L, Hong Y, Guan J, Stankus JJ, El-Kurdi MS, Wagner WR, et al. A bilayered elastomeric scaffold for tissue engineering of small diameter vascular grafts. Acta Biomaterialia. 2010;6:110-22.

30. Soletti L. Development of a Stem Cell-Based Tissue Engineered Vascular Graft [PhD thesis]. Pittsburgh, PA: University of Pittsburgh; 2008.

31. Soletti L, Nieponice A, Guan J, Stankus JJ, Wagner WR, Vorp DA. A seeding device for tissue engineered tubular structures. Biomaterials. 2006; 27:4863-70.

32. Shinoka T. What is the best material for extracardiac Fontan operation? J Thorac Cardiovasc Surg. 2017;153:1551-2.

33. Fukunishi T, Best CA, Sugiura T, Opfermann J, Ong CS, Shinoka T, et al. Preclinical study of patient-specific cell-free nanofiber tissue-engineered vascular grafts using 3-dimensional printing in a sheep model. J Thorac Cardiovasc Surg. 2017; 153:924-32.

34. Sugiura T, Matsumura G, Miyamoto S, Miyachi H, Breuer CK, Shinoka T. Tissue-engineered vascular grafts in children with congenital heart disease: intermediate term follow-up. Semin Thorac Cardiovasc Surg. 2018;30:175-9.

35. McAllister TN, Maruszewski M, Garrido SA, Wystrychowski W, Dusserre N, Marini A, et al. Effectiveness of haemodialysis access with an autologous tissue-engineered vascular graft: a multicentre cohort study. Lancet. 2009;373: 1440-6.

36. Dahl SL, Kypson AP, Lawson JH, Blum JL, Strader JT, Li Y, et al. Readily available tissue-engineered vascular grafts. Sci Transl Med. 2011;3:68-9.

37. Hashi CK, Derugin N, Janairo RRR, Lee R, Schultz D, Lotz J, et al. Antithrombogenic modification of small-diameter microfibrous vascular grafts. Arterioscler Thromb Vasc Biol. 2010;30:1621-7.

38. Hibino N, Yi T, Duncan DR, Rathore A, Dean E, Naito Y, et al. A critical role for macrophages in neovessel formation and the development of stenosis in tissueengineered vascular grafts. Faseb J. 2011;25:4253-63.

39. Basu J, Genheimer CW, Guthrie KI, Sangha N, Quinlan SF, Bruce AT, et al. Expansion of the human adipose-derived stromal vascular cell fraction yields a population of smooth muscle-like cells with markedly distinct phenotypic and functional properties relative to mesenchymal stem cells. Tissue Eng Part C Methods. 2011;17:843-60.

40. Rodriguez J, Pratta AS, Abbassi N, Fabre H, Rodriguez F, Debard C, et al. Evaluation of three devices for the isolation of the stromal vascular fraction from adipose tissue and for ASC culture: a comparative study. Stem Cells Int. 2017;2017: 9289213.

Key Words: arterial, fat, lipoaspirate, phenotype, polymer, remodeling, stromal vascular fraction, translation 


\section{SUPPLEMENTAL MATERIAL. ADDITIONAL} IMMUNOFLUORESCENT CHEMISTRY STAINING

Further IFC analysis of our seeded constructs was done for additional SVF cell markers. These markers included 1:100 dilutions of rabbit anti-human CD14 (ab183322, Abcam, Cambridge, UK), rabbit anti-human CD45 (ab10558, Abcam), and CD105 (ab1695450, Abcam) to evaluate the presence of monocytes, leukocytes, or additional progenitor cell populations, respectively. All IFC staining included a primary delete for a negative control and an additional isotype control (ab172730, Abcam). Seeded constructs were then incubated with 1:1000 dilutions of FITC-conjugated goat anti-rabbit IgG secondary antibody (611-1202, Rockland Inc, Pottstown, Pa) and then counterstained with DAPI (B2883, Sigma-Aldrich, St Louis, Mo) to mark cell nuclei. Imaging was conducted on a Nikon Eclipse 90i microscope (Nikon, Tokyo, Japan) where multiple $10 \times \mathrm{im}-$ ages were taken for each stained scaffold section around the circumference covering approximately $90 \%$ of the full ring without overlap.

IFC analysis of the seeded SVF/ADMSC phenotypes showed little or no presence of CD14 or CD45, indicating such immune cells were at most minimally adherent to the PEUU scaffolds (Figure E1). Results show little to no attachment of immune cells to our scaffolds and little presence of CD105 positive cells, although CD105 did trend to increase at $\mathrm{P} 4$, but it was not significant.

\section{IMAGEJ MACRO ANALYSIS}

First, each multi-channel (ie, colored) $10 \times$ image was imported and the DAPI channel was extracted. A Gaussian blur was applied to the image and subtracted from the original to eliminate background noise and develop more sharply defined particles. An Otsu threshold was then applied to form a binary image used in subsequent counting steps. The particles were then eroded and subsequently dilated to eliminate imaging artifacts. Finally, a watershed transformation was applied before counting that separated touching circular objects. Greater than $99 \%$ accuracy of the cell counting macro was validated by comparison with manual counting by 3 independent observers.

Non-nuclear stains were counted through visual inspection, because these markers were not fit to clearly defined elliptical shapes. Cells exhibiting a specific cell marker (CD90, CD31, or CD34, and CD14, CD45, or CD105) were counted by hand again by 3 independent observers and were within $2 \%$ of each other on average. Each specimen included measurements from images taken from multiple cryosections and then averaged for the sample.

\section{REMODELING CLASSIFICATIONS}

A fully remodeled TEVG explant was defined as one that was observed to be patent by angiography, demonstrated neotissue formation on gross inspection, and contained defined vascular components in both IFC and histology. A partially remodeled TEVG explant was defined as one that was observed to be patent by angiography but did not demonstrate neotissue formation on gross inspection or contained some vascular components in IFC but not in any defined manner. An occluded TEVG explant was defined as one that was not observed to be patent by angiography or through gross inspection. 


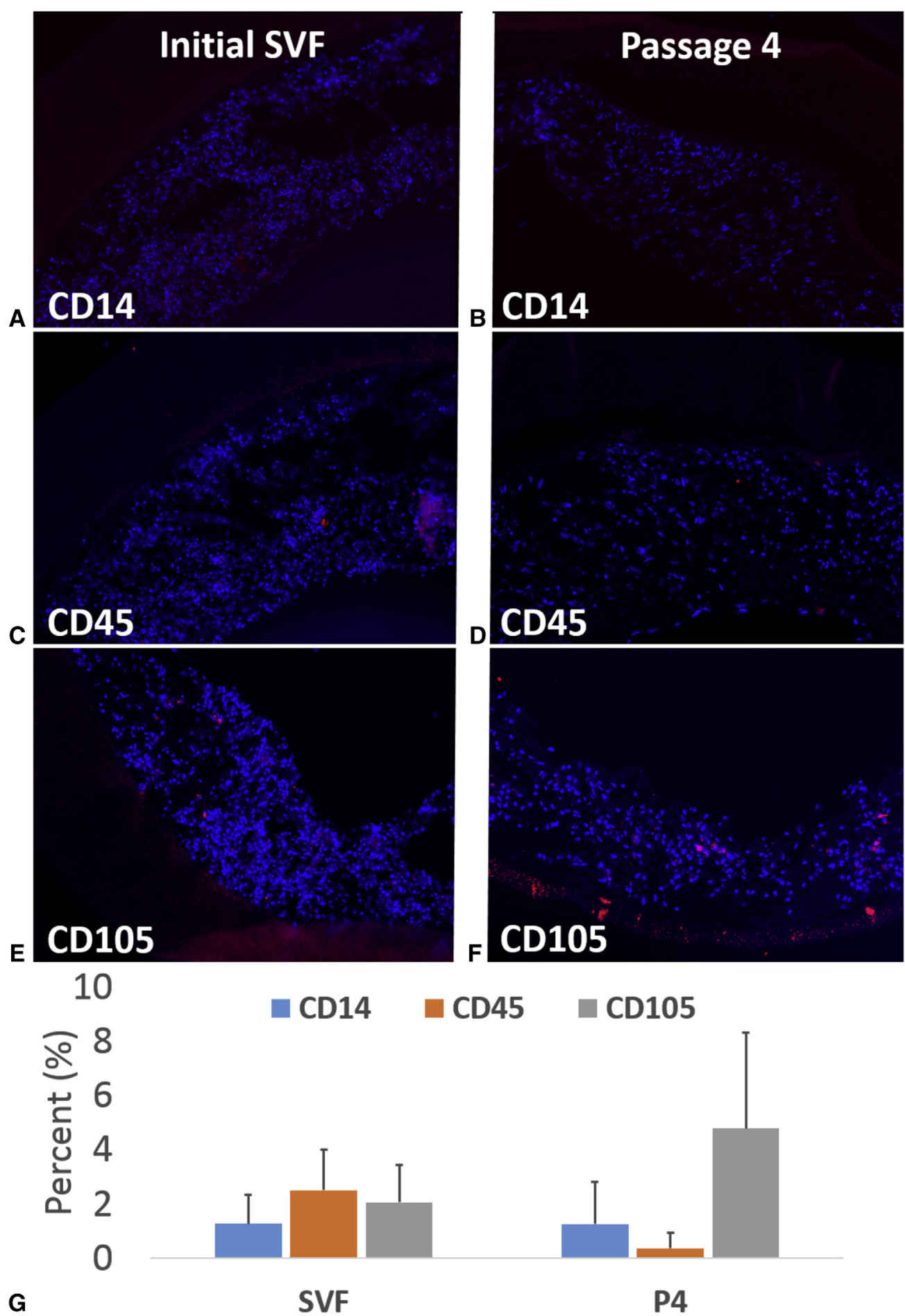

FIGURE E1. Representative IFC images depicting positive staining of cells for CD14 (A and B) and CD45 (C and D) showing little to no attachment of immune cells to our scaffolds from SVF or at passage 4 (P4). A small population of SVF cells stained positively for CD105 (E and F) and increased positive staining at P4. G, IFC analysis of percentage of adherent cells positive for cell markers CD14 (monocyte), CD45 (leukocyte), and CD105 (progenitor marker) seeded onto PEUU scaffolds with cells from fresh SVF and P4, both given a 48-hour dynamic culture period. SVF, Stromal vascular fraction. 


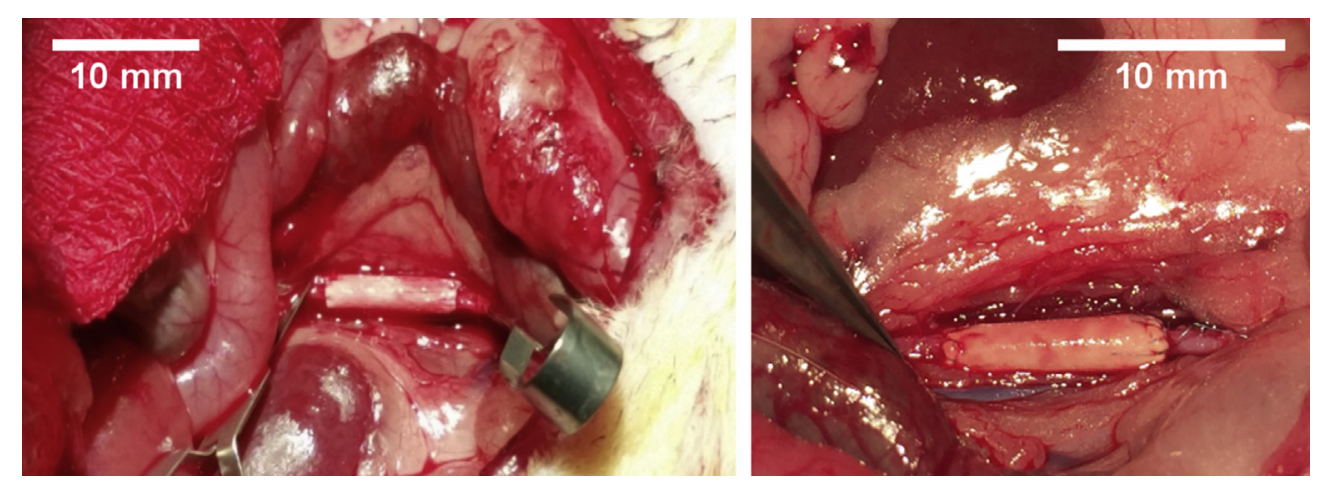

FIGURE E2. Left: Example of SVF-seeded construct in the interpositional abdominal aortic position before clamp removal. Right: Implanted construct immediately after suturing in place and having blood flow resumed. 